\title{
Grape Seed Proanthocyanidins play the roles of radio- protection on Normal Lung and radiosensitization on Lung Cancer via differential regulation of the MAPK Signaling Pathway
}

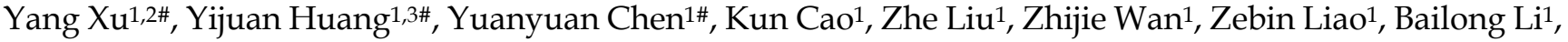 \\ Jianguo Cui ${ }^{1}$, Yanyong Yang ${ }^{1}$, Xiaohua $\mathrm{Xu}^{4}{ }^{\bowtie}$, Jianming Cai ${ }^{\bowtie}$ and Fu Gao ${ }^{\circledR}$ \\ 1. Department of Radiation Medicine, Faculty of Naval Medicine, Naval Medical University, 800 Xiangyin Road, Shanghai, China, 200433 \\ 2. Department of Radiology, Xizang Military General Hospital, 66 Niangre North Road, Lhasa City, Tibet, China, 850000. \\ 3. Department of Radiology, First Hospital of Jiaxing, Affiliated Hospital of Jiaxing University, 1882 Zhonghuan South Road, Jiaxing, Zhejiang, 314000. \\ 4. Department of Nuclear Radiation, Tongji University Affiliated Shanghai Pulmonary Hospital, 507 Zhengmin Road, Shanghai, China, 200433 \\ \#These authors contributed equally to this work. \\ $\triangle$ Corresponding authors: Fu Gao, Department of Radiation Medicine, Faculty of Naval Medicine, Naval Medical University, 800 Xiangyin Road, Shanghai, China, 200433. \\ E-mail: gaofusmmu@163.com; Jianming Cai, Department of Radiation Medicine, Faculty of Naval Medicine, Naval Medical University, 800 Xiangyin Road, Shanghai, China, \\ 200433. E-mail: caijianming882003@aliyun.com; Xiaohua Xu, Department of Nuclear Radiation, Tongji University Affiliated Shanghai Pulmonary Hospital, 507 Zhengmin \\ Road, Shanghai, China, 200433. E-mail: xiaohua621218@sina.com.
}

(C) The author(s). This is an open access article distributed under the terms of the Creative Commons Attribution License (https://creativecommons.org/licenses/by/4.0/). See http://ivyspring.com/terms for full terms and conditions.

Received: 2020.06.26; Accepted: 2021.02.03; Published: 2021.03.15

\begin{abstract}
Radiation-induced lung injury (RILI) is a common serious complication and dose-limiting factor caused by radiotherapy for lung cancer. This study was to investigate radioprotective effects of grape seed proanthocyanidins (GSP) on normal lung as well as radiosensitizing effects on lung cancer. In vitro, we demonstrated radioprotective effects of GSP on normal alveolar epithelial cells (MLE-12 and BEAS/2B) and radiosensitizing effects on lung cancer cells (LLC and A549). In vivo, we confirmed these two-way effects in tumor-bearing mice. The results showed that GSP inhibited tumor growth, and played a synergistic killing effect with radiotherapy on lung cancer. Meanwhile, GSP reduced radiation damage to normal lung tissues. The two-way effects related to the differential regulation of the MAPK signaling pathway by GSP on normal lung and lung cancer. Moreover, GSP regulated secretion of cytokines IL-6 and IFN- $\gamma$ and expression of p53 and Ki67 on normal lung and lung cancer. Our findings suggest that GSP is expected to be an ideal radioprotective drug for lung cancer patients who are treated with radiotherapy.
\end{abstract}

Key words: Proanthocyanidins; lung cancer; radiosensitization; radioprotection; MAPK

\section{Introduction}

Lung cancer ranks first in morbidity and mortality among malignant tumors [1], and radiotherapy is one of the main treatments for lung cancer. The incidence of radiation-induced lung injury (RILI) in patients with lung cancer after chest radiotherapy is $15 \%$ to $40 \%$ [2]. The occurrence of RILI severely limits the dose of radiotherapy in the target area. Some patients even need to discontinue radiotherapy due to the occurrence and progressive exacerbation of RILI. Severe radiation-induced pulmonary fibrosis can even lead to death of patients [3, 4]. Therefore, the protection of RILI caused by radiotherapy has extremely important medical significance. The main reason that some currently known radioprotective agents cannot be used in the clinic is that they prevent radiation damage to normal tissues and also have a radioprotective effect on tumor tissues, reducing the effect of radiotherapy [5-8].

Proanthocyanidin has obvious free radical scavenging ability, and its antioxidant activity in the body is 20 times that of VC and 50 times that of VE [9, 10]. Free radicals play important roles in injuries induced by ionizing radiation. Our previous experiments have found that grape seed pro- 
anthocyanidins (GSP) have significant radioprotective effects on radiation pneumonitis and pulmonary fibrosis [11, 12]. In addition, proanthocyanidins also have anti-tumor effects. The study of breast cancer [13], colorectal cancer [14], liver cancer [15], gastric cancer [16], oral squamous cell carcinoma [17], bladder cancer [18], skin cancer [19], kidney cancer [20], prostate cancer [21] and lung cancer [22] reported the obvious anti-tumor effects of proanthocyanidins. Therefore, when GSP is used for lung cancer patients who are treated with radiotherapy, it protects normal lung tissue against ionizing radiation and meanwhile it may enhance the killing effect of radiotherapy for lung cancer, which means that GSP is expected to be an ideal radioprotective drug for lung cancer patients who are treated with radiotherapy.

Based on different effects of proanthocyanidins on normal lung and lung cancer, this study conducted a preliminary discussion on its mechanism. Mitogenactivated protein kinase (MAPK) plays important roles in regulating proliferation, differentiation and apoptosis of cells [23]. The MAPK/ERK signaling pathway is mainly related to cell proliferation and differentiation, while the MAPK/JNK and $\mathrm{MAPK} / \mathrm{p} 38$ pathways are closely related to apoptosis of cells [23]. Activation of MAPK/ERK signaling pathway promotes the growth of prostate cancer [24], cervical cancer [25], thyroid cancer [26], and breast cancer [27]. MAPK/ERK-induced epithelialmesenchymal transition promotes migration and metastasis of lung cancer cells [28]. However, Blocking the MAPK/ ERK signaling pathway suppresses proliferation of human ovarian cancer cells [29] and prostate cancer cells [30]. Unlike the MAPK/ERK pathway, activating the MAPK/JNK or MAPK/p38 pathway induces apoptosis of tumor cells, such as prostate cancer cells [24], breast cancer cells [31, 32], gastric cancer cells [33], and lung cancer cells [34]. Anthocyanins induce apoptosis of colon cancer cells [35] and leukemic cells [36] by activating the MAPK/JNK or MAPK/p38 pathway. However, for normal cells, down-regulating the MAPK pathway protect them when they are physically or chemically damaged. Anthocyanins also protect normal cells by down-regulating the MAPK signaling pathway. Grape seed proanthocyanidins inhibit the activation of MAPK signaling pathway mediated by UV-induced oxidative stress in human epidermal keratinocytes [37]. Grape seed proanthocyanidins played a similar effect in UV-exposed mouse skin when given to animals in the diet [38]. Anthocyanins protect the retinal pigment epithelial cells from damage by suppressing the MAPK signaling pathway [39]. Moreover, anthocyanins attenuate osteoarthritis $[40,41]$, encephalitis $[42,43]$, and skin inflammation
[44] by inhibiting the MAPK signaling pathway. In this study, we investigated dual role of grape seed proanthocyanidins in normal lung and lung cancer after ionizing radiation, and found that the diverse regulation of MAPK signaling pathway account for the underlying mechanism.

\section{Materials and methods}

\section{Cell culture and GSP treatment}

All cells were from American Type Culture Collection. LLC (Mice Lewis lung cancer cells) and A549 cells (human non-small cell lung cancer cells) were cultured in DMEM medium and BEAS-2B (Human bronchial epithelial cells) and MLE-12 cells (mice lung epithelial cells) were cultured in RPMI 1640 medium. Both the DMEM and RPMI 1640 medium contained $10 \%$ fetal calf serum. Cell incubator kept at $37{ }^{\circ} \mathrm{C}$ with 5\% CO2 and 95\% humidity.

Grape seed proanthocyanidins (GSP) was obtained from Tianjin Peak Natural Products Research and Development co. LTD. (Tianjin, China). One hour before ionizing radiation, cells were pretreated with or without GSP-containing PBS. Twenty-four hours after treatment, cells were transferred to normal DMEM of RPMI 1640 medium.

\section{CCK-8 assay, colony formation assay and flow cytometric analysis}

Cell viability was detected by CCK-8 assay (Cell Counting Kit-8; Dojindo, Kumamoto, Japan). Cell proliferation was detected by Colony formation assay as previous research [12]. Cell apoptosis was detected by flow cytometric analysis using an Apoptosis Detection Kit (Invitrogen, Carlsbad, California, USA).

\section{Intracellular ROS measurement}

The anti-oxidant (NAC) was used as a positive control to detect the scavenging effect of GSP on free radicals after irradiation. One hour before irradiation, GSP was given at a concentration of $20 \mathrm{ug} / \mathrm{ml}$ and $\mathrm{NAC}$ at a concentration of $10 \mathrm{mmol} / \mathrm{L}$. Reactive Oxygen Species Fluorogenic Probe (Cat. No S0033; Beyotime; China) was used for measurement of intracellular ROS of A549 and BEAS-2B cells.

\section{Mice and GSP treatments}

C57BL/6 6-week-old male mice were purchased from Shanghai Ling Chang biological technology co., LTD. All the experiments associated with mice were approved by the Laboratory Animal Center of Naval Medical University, Shanghai. Mice were used and randomly divided into six groups: two groups without lung cancer and other four groups with lung cancer. We injected $1 \times 10^{6}$ Lewis lung carcinoma cells 
(LLC, ATCC) mixed with Matrigel (Matrigel: Medium, a ratio of 1:1, $25 \mu$ l total) into the left upper lobe of the mice to establish the lung cancer model. The lung cancer mice were divided into four groups, including a non-irradiated group, a GSP treatment group, an irradiation group and an irradiation with GSP treatment group. The lung cancer-free mice were divided into two groups, including a sham operation group and a simple Matrigel injected group. GSP (2 $\mathrm{mg} / \mathrm{ml}$ ) was delivered through drinking after LLC cells injected.

\section{Lung extraction and pathological staining}

The left upper lobe lungs were resected and weighed at different times. The left upper lobe lungs, with or without cancers, were then used for pathological analysis and western blot analysis. HE staining and immunofluorescence staining was performed as previously described [45]. Anti-P53 (1:500) and anti-Ki67 (1:500) antibodies were from Cell Signaling Tech, China.

After the upper lobe of the left lung of the mouse was embedded in paraffin, it was sectioned and stained every $0.5 \mathrm{~mm}$ with a microtome, and the largest cross section of the tumor was taken as the experimental result. The cross-sectional area of the tumor on the $4^{\text {th }}, 7^{\text {th }}, 11^{\text {th }}, 14^{\text {th }}, 15^{\text {th }}, 16^{\text {th }}$ and $18^{\text {th }}$ day after irradiation was compared with the cross-sectional area of the tumor on the 1st day after irradiation in each group, and the value obtained was used to compare the groups. In the same way, the weight changes of the upper left lung lobe between the groups were compared.

\section{ELISA assay}

ELISA kits (Westang Tech., Shanghai, China) were used to detect the serum levels of IL-6 and IFN- $\gamma$.

\section{Western blot analysis}

Proteins from cells and tissues were extracted by ProtecJETTM Mammalian Cell Lysis Reagent (Fermentas, Vilnius, Baltic, Lithuania). MAPK related antibodies (1:1000) were provided by Abcam Corporation. Other antibodies including the secondary antibody (1:1000) were provided by Cell Signaling Technology Corporation.

\section{Irradiation}

LLC, A549, MLE-12 and BEAS-2B cells were exposed to ${ }^{60} \mathrm{Co}$ in the radiation center (Naval Medical University, Shanghai) with a dose of $8 \mathrm{~Gy}$ at a dose rate of $1 \mathrm{~Gy} / \mathrm{min}$. Local chest of all radiated mice were exposed to ${ }^{60} \mathrm{Co}$ with a dose of $25 \mathrm{~Gy}$ at a dose rate of 1 Gy/min.

\section{Statistical analysis}

Data were expressed as mean \pm SD of three independent experiments and calculated using oneway ANOVA (Prism version 6.0 software). StudentNewman-Keuls post-hoc test was used to determine variance between groups. The difference between the groups was considered statistically significant when $P$ $<0.05$.

\section{Results}

\section{Grape seed proanthocyanidins (GSP) sensitized lung cancer cells to ionizing radiation (IR) while alleviated radiation damage to normal lung cells}

In CCK8 assay, no toxicity was found in both cancer cells (LLC and A549) and normal cells (MLE-12 and BEAS-2B) treated with GSP at the concentration less than $20 \mathrm{ug} / \mathrm{ml}$ (Figure 1A). And at the concentration of $20 \mathrm{ug} / \mathrm{ml}$, no toxicity was found until 24 hours after administration (Figure 1B). In flow cytometry, compared with IR group, GSP treatment significantly decreased apoptosis in MLE-12 cells and BEAS-2B cells, while increased apoptosis in A549 cells (Figure 1C). The colony formation assay confirmed that GSP treatment significantly improved the viability of MLE-12 cells and BEAS-2B cells after irradiation, and reduced the viability of A549 after irradiation (Figure 1D). Although no significant difference was found in LLC cells at a concentration of $20 \mathrm{ug} / \mathrm{ml}$ in flow cytometry analysis, clone formation assays showed that GSP reduced the viability of LLC cells after irradiation. In brief, we found that GSP sensitized lung cancer cells to IR while reducing radiation damage to normal lung cells.

\section{Suppressive and radiosensitive potential of GSP on tumor in vivo}

To mimic the microenvironment of lung cancer, we injected LLC cells into the left upper lobe of C57BL/ 6 mice. We found that IR, GSP, and IR+GSP reduced tumor volume in tumor-bearing mice (Figure $2 \mathrm{~A}, 2 \mathrm{~B})$, reduced the weight of the lung lobe where the tumor was located (Figure 2C), improved the adverse effect of lung cancer on body weight (Figure 2D) and increased survival (Figure 2E). The effect of IR+GSP was stronger than IR and GSP, indicating that GSP had the effect of inhibiting tumor growth, and played a synergistic effect with IR to kill tumor cells.

\section{GSP attenuated inflammation of normal lung tissue and promoted apoptosis of tumor after irradiation}

From the HE stained sections, inflammation was found in normal lung tissue surrounding the tumor 
(Figure 3A). GSP treatment markedly attenuated the inflammation. After local chest ionizing radiation, a large number of inflammatory cells accumulated in normal lung tissue, and the extracellular matrix was excessively deposited. The normal structure of the alveoli was destroyed, with thickened alveolar wall. However, GSP treatment significantly alleviated these changes.

Immunohistochemical staining showed that ionizing radiation increased the expression of P53 in both normal lung tissues and lung cancer tissues, but GSP reduced the increment of P53 expression in normal lung tissues (Figure 3B) while further increase the expression of P53 in lung cancer tissues (Figure 3C). In addition, we found that GSP down-regulated the expression of Ki67 in lung cancer tissues while it had no effect on normal lung tissues.

\section{GSP regulated secretion of cytokines IL-6 and IFN-Y after ionizing radiation}

Tumor-bearing mice had significantly higher IL-6 levels and lower IFN- $\gamma$ levels compared with the normal mice (Figure 4A, 4B). The serum of tumor-bearing mice after local chest irradiation was detected, and the serum IL-6 level was significantly increased 6 hours after irradiation. At 12 hours after irradiation, the serum IL-6 level in IR+GSP mice was significantly lower than that of IR mice, while IFN- $\gamma$ levels were significantly increased (Figure 4C, 4D).

\section{GSP reduced ROS levels in both cancer cells A549 and normal cells BEAS-2B}

The anti-oxidant (NAC) was used as a positive control to detect the scavenging effect of GSP on free radicals after irradiation. The intracellular ROS level of A549 and BEAS-2B cells were detected after irradiation by Reactive Oxygen Species Fluorogenic Probe. We found that GSP treatment significantly reduced ROS levels in both cancer cells A549 (Figure 5A) and normal cells BEAS-2B (Figure 5B).

\section{GSP differentially regulated MAPK signaling pathways in lung cancer cells/tissues and normal lung cells/tissues}

In lung cancer cells A549, GSP significantly increased the expression of p-JNK, p-P38 protein before and after ionizing radiation, and the value of Bax/Bcl-2 after irradiation, and had less effect on p-ERK protein (Figure 6A). In normal lung epithelial cells BEAS-2B, GSP reduced the expression of p-JNK, p-P38, and p-ERK proteins of MAPK family after ionizing radiation, and reduced the expression of Bax and value of Bax/ Bcl-2 (Figure 6B).
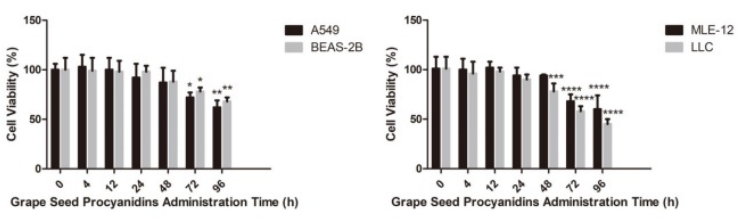

D
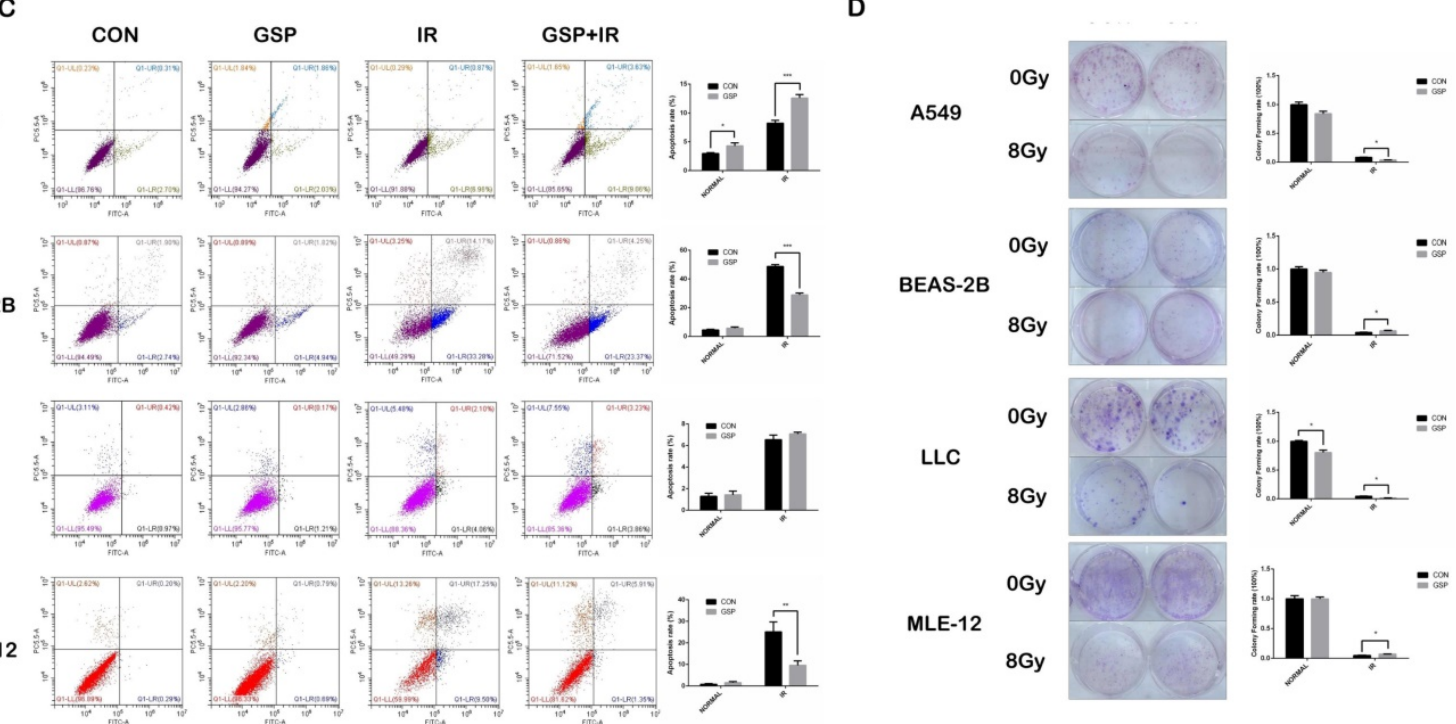

Figure 1. Grape seed proanthocyanidins (GSP) sensitizes lung cancer cells LLC and A549 to ionizing radiation (IR) while alleviates radiation damage to normal lung cells MLE-12 and BEAS-2B. (A) Cytotoxicity of different concentrations of GSP on lung cancer cells and normal lung cells. In CCK 8 assay, no toxicity is found in both cancer cells (LLC and A549) and normal cells (MLE-12 and BEAS-2B) treated with GSP at the concentration less than 20 ug/ml. (B) Cytotoxicity of GSP on lung cancer cells and normal lung cells at different administration times. At the concentration of $20 \mathrm{ug} / \mathrm{ml}$, no toxicity is found until 24 hours after administration. (C) In flow cytometry, compared with IR group, GSP treatment significantly decreases apoptosis in MLE-12 cells and BEAS-2B cells, while increases apoptosis in A549 cells. (D) The colony formation assay shows that GSP treatment significantly improves the viability of MLE- 12 cells and BEAS-2B cells after irradiation, and reduces the viability of A549 after irradiation. LLC, A549, MLE-12 and BEAS-2B cells were exposed to ${ }^{60} \mathrm{Co}$ with a dose of $8 \mathrm{~Gy}$ at a dose rate of $1 \mathrm{~Gy} / \mathrm{min}$. Data were presented as mean $\pm \mathrm{SD}(\mathrm{n}=3)$. $* \mathrm{P}<0.05, * * \mathrm{P}<0.01, * * * \mathrm{P}<0.001, * * * * \mathrm{P}<0.0001$. 
A
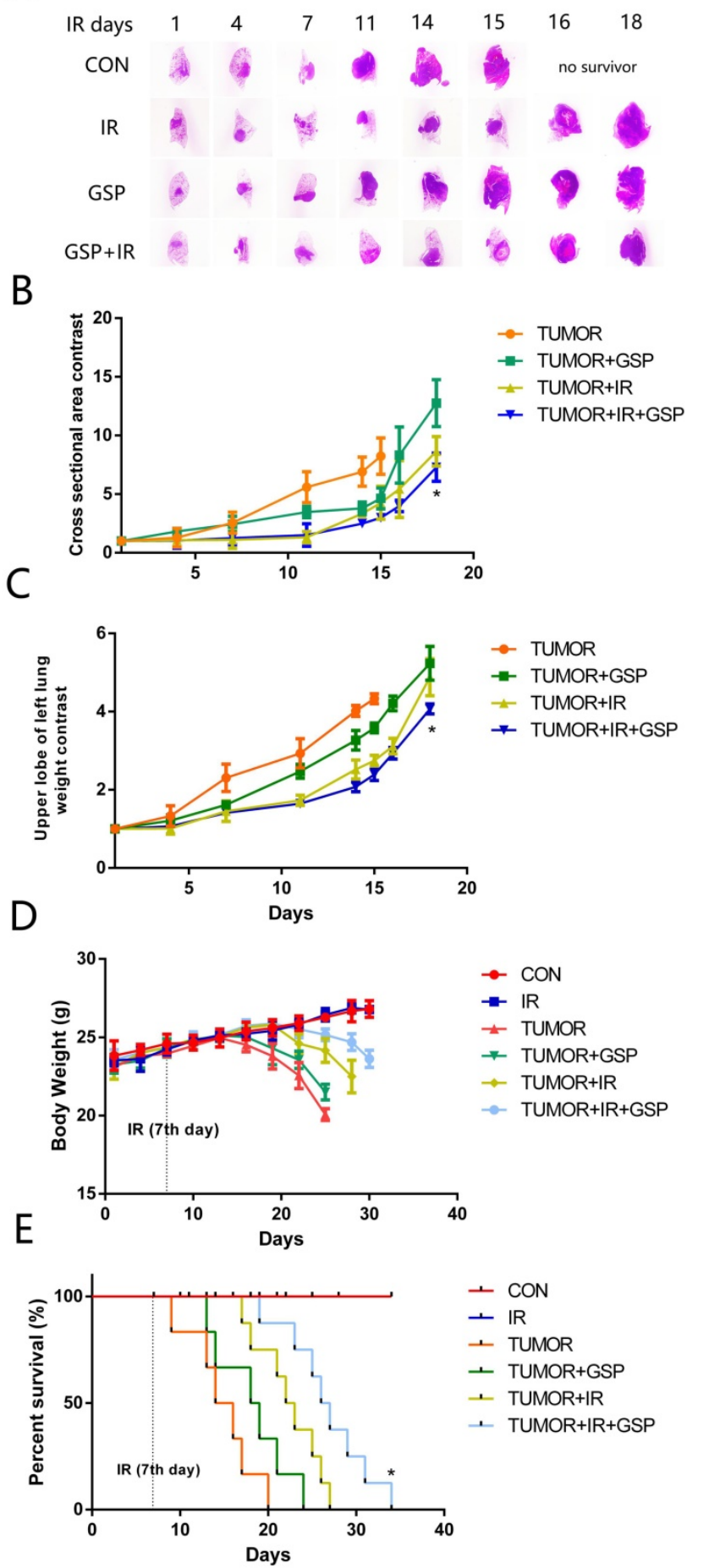

Figure 2. Suppressive and radiosensitive potential of GSP on tumor in vivo. (A) Representative images of HE stained sections of lung tissues with tumor. The largest cross section of the tumor is taken as the experimental result. IR, GSP, and IR+GSP reduce tumor size in tumor-bearing mice. (B) The cross-sectional area of the tumor on the $4^{\text {th }}, 7^{\text {th }}, 11^{\text {th }}, 14^{\text {th }}, 15^{\text {th }}, 16^{\text {th }}$ and $18^{\text {th }}$ day after irradiation is compared with the cross-sectional area of the tumor on the 1st day after irradiation in each group, and the value obtained is used to compare the groups. The results show that IR + GSP has the most obvious inhibitory effect on tumor. (C) In the same way, the weight changes of the upper left lung lobe between the groups are compared. IR, GSP, and IR+GSP reduce the weight of the lung lobe where the tumor is located. (D) IR, GSP, and $I R+G S P$ improve the adverse effect of lung cancer on body weight, most significant in $I R+G S P$ group. (E) IR, GSP, and IR+GSP improve the survival rate of tumor-bearing mice, most significant in IR+GSP group. Local chest of all radiated mice were exposed to ${ }^{60} \mathrm{Co}$ with a dose of $25 \mathrm{~Gy}$ at a dose rate of $1 \mathrm{~Gy} / \mathrm{min}$. GSP $(2 \mathrm{mg} / \mathrm{ml})$ was delivered through drinking after LLC cells injected. Data were presented as mean $\pm S D(n=3)$. $* \mathrm{P}<0.05$, ** $\mathrm{P}<0.01, * * * \mathrm{P}<0.001, * * * * \mathrm{P}<0.0001$
In tumor-bearing mice, ionizing radiation increased expression of $\mathrm{p}$-JNK, p-P38, and p-ERK proteins of MAPK family in normal lung tissues, while GSP reduced the increments. In lung cancer tissues, GSP activated the expression of $\mathrm{p}$-JNK protein (Figure 7A). In normal mice, the expression of $\mathrm{p}-\mathrm{JNK}$, p-P38, and p-ERK proteins of MAPK family in the lung tissue was significantly reduced when GSP was given in diet, but the protein expression gradually increased over the feeding time (Figure 7B).

\section{Discussion}

Radiotherapy is one of the main treatments for lung cancer. Radiation-induced lung injuries (RILI) are common serious complications and dose limiting factors of radiotherapy for lung cancer. One of the main reasons for limiting the clinical application of radioprotective drugs for lung cancer patients who are treated with radiotherapy is that existing radioprotective drugs have protective effects on both normal lung tissue and lung cancer tissue. In this study, a model of lung cancer-bearing mice was established, and the results showed that grape seed proanthocyanidins (GSP) had a radioprotective effect on normal lung tissue and a radiosensitizing effect on lung cancer tissue. Therefore, GSP is expected to be a very ideal radioprotective drug for lung cancer patients who are treated with radiotherapy.

Proanthocyanidins have obvious antioxidative ability, and have good protective effect on oxidative stress related diseases. Proanthocyanidins have protective effects on UV-B-induced oxidative damage to keratinocytes through activation of Nfr2 signaling [46]. Proanthocyanidins protect renal tubular cell against apoptosis induced by oxidative stress in mice [47]. Proanthocyanidins significantly increase the content of superoxide dismutase and peroxidase in liver tissues after ionizing radiation, thereby significantly protecting the liver tissues of exposed rats [48]. Other researchers reported that proanthocyanidins increase the number of peripheral blood lymphocytes in 6Gy-irradiated rats and reduce the damage to the antioxidant system caused by free radicals [49]. Our previous experiments demonstrated that proanthocyanidins have radioprotective effects on RILI in normal mice [12]. This study confirmed the protective effect of proanthocyanidins on RILI in a lung cancer-bearing mouse model. In addition, proanthocyanidins also played a synergistic killing effect on lung cancer tissues, improving the radiotherapy effect. Compared with the IR and GSP groups, the IR + GSP group had better cancer suppression effect and survival rate. The results of $\mathrm{HE}$ and immunofluorescence staining showed that GSP reduced the inflammatory response of normal lung tissues, while 
enhancing the killing effect of radiotherapy on lung cancer. The antitumor effects of proanthocyanidins have been reported. The study of breast cancer [13], colorectal cancer [14], liver cancer [15], gastric cancer [16], oral squamous cell carcinoma [17], bladder cancer [18], skin cancer [19], kidney cancer [20], prostate cancer [21] and lung cancer [22] reported the obvious antitumor effects of proanthocyanidins. This study revealed that proanthocyanidins protected normal lung tissue against ionizing radiation and sensitized lung cancer tissues to ionizing radiation simultaneously. We observed similar results in experiments in vitro. Through flow cytometry and colony formation analysis, we found that proanthocyanidins had radiosensitizing effects on lung cancer cells LLC and A549, but had radioprotective effect on normal cells MLE-12 and BEAS-2B.

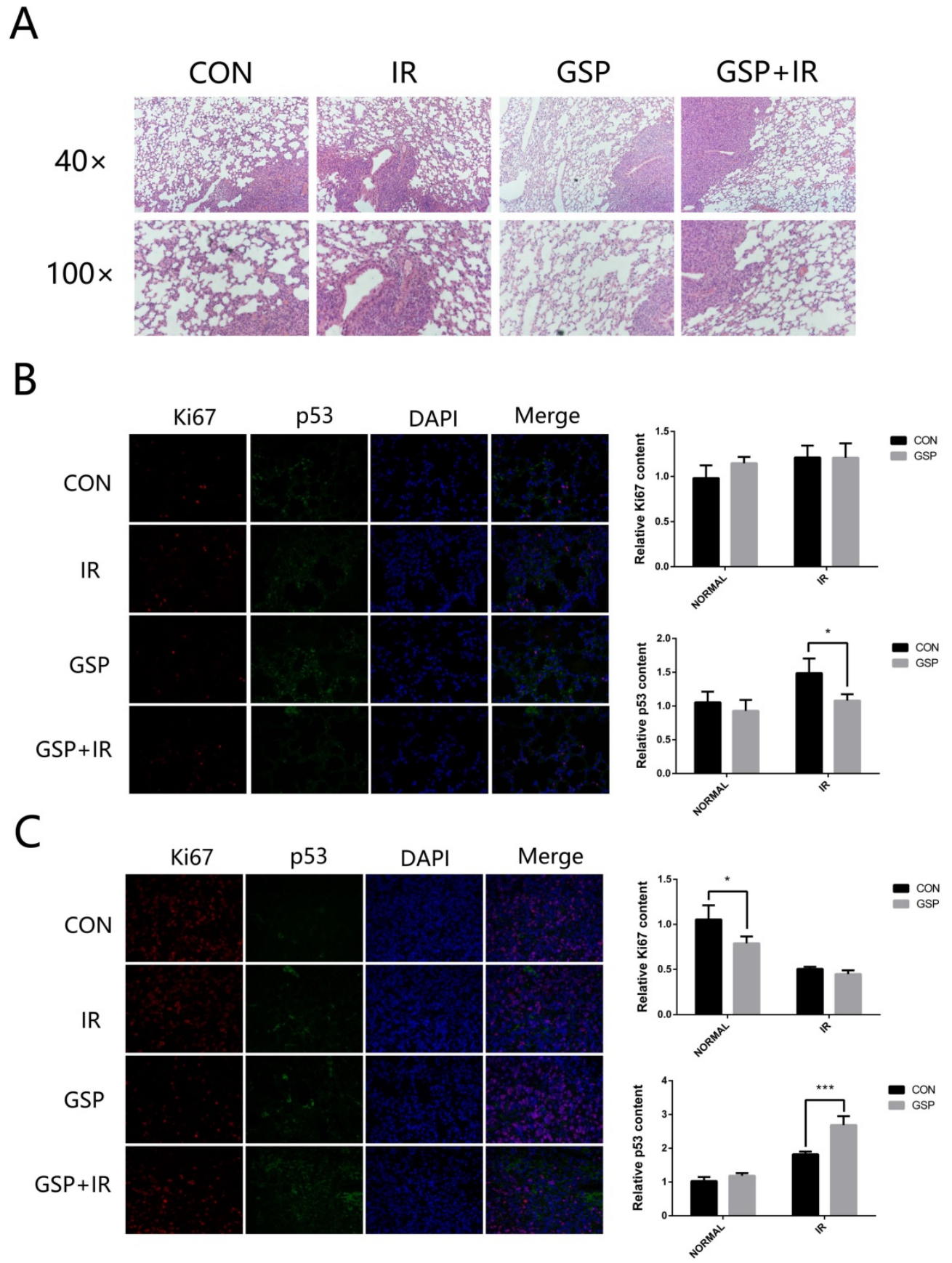

Figure 3. GSP attenuates inflammation of normal lung tissues and promotes apoptosis of tumor after irradiation. (A) Representative images of HE stained sections of lung tissues with tumor. GSP treatment markedly attenuates the inflammation in normal lung tissue surrounding the tumor. After local chest ionizing radiation, a large number of inflammatory cells accumulate in normal lung tissue, and the extracellular matrix is excessively deposited. The normal structure of the alveoli is destroyed, with thickened alveolar wall. However, GSP treatment significantly alleviates these changes. (B) Representative images and a quantification of Ki67 and P53 expressions in normal lung tissues by immunohistochemical staining. lonizing radiation increases the expression of P53 in normal lung tissues, while GSP reduces the increment of P53 expression. GSP has no effect on the expression of Ki67 in normal lung tissues. (C) Representative images and a quantification of Ki67 and P53 expressions in tumor by immunohistochemical staining. lonizing radiation increases the expression of P53 in lung cancer tissues, and GSP further increases the expression of P53. In addition, GSP down-regulates the expression of Ki67 in lung cancer tissues. Data were presented as mean $\pm S D(n=3)$. *P<0.05, **P $<0.01, * * * P<0.001$, **** $P<0.0001$. 
A

IL-6

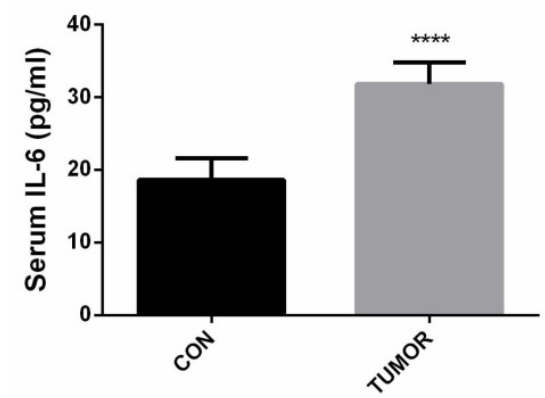

C

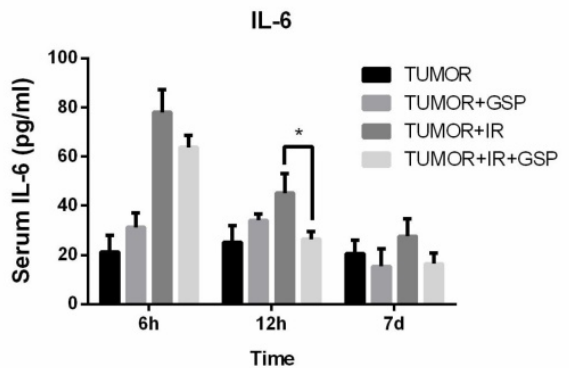

B

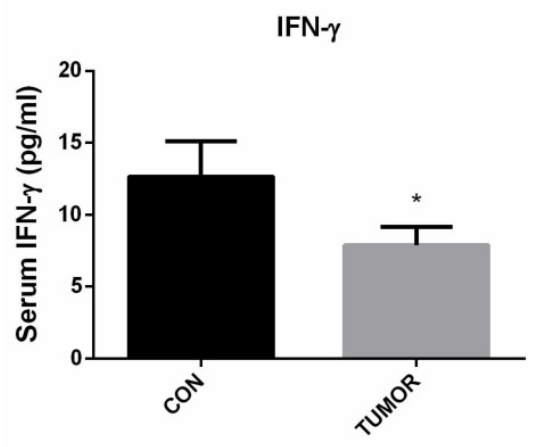

D

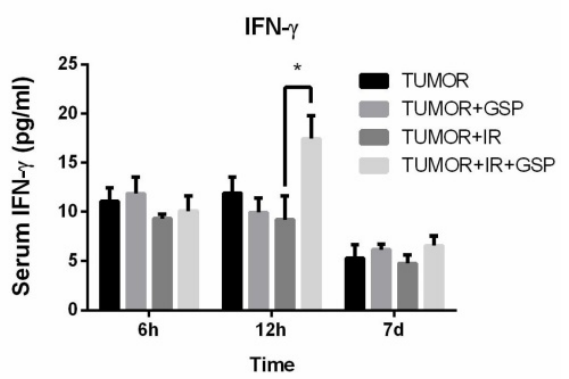

Figure 4. GSP regulates secretion of cytokines IL-6 and IFN-y after ionizing radiation. Compared with the normal mice, tumor-bearing mice have significantly higher IL-6 levels (A) and lower IFN-Y levels (B). At 12 hours after irradiation, the serum IL-6 level in the IR+GSP mice is significantly lower than that of IR mice (C), while IFN-Y levels are significantly increased (D). Data were presented as mean \pm SD $(n=3)$. $* P<0.05$, **P $<0.01$, *** $P<0.001$, $* * * * P<0.0001$.

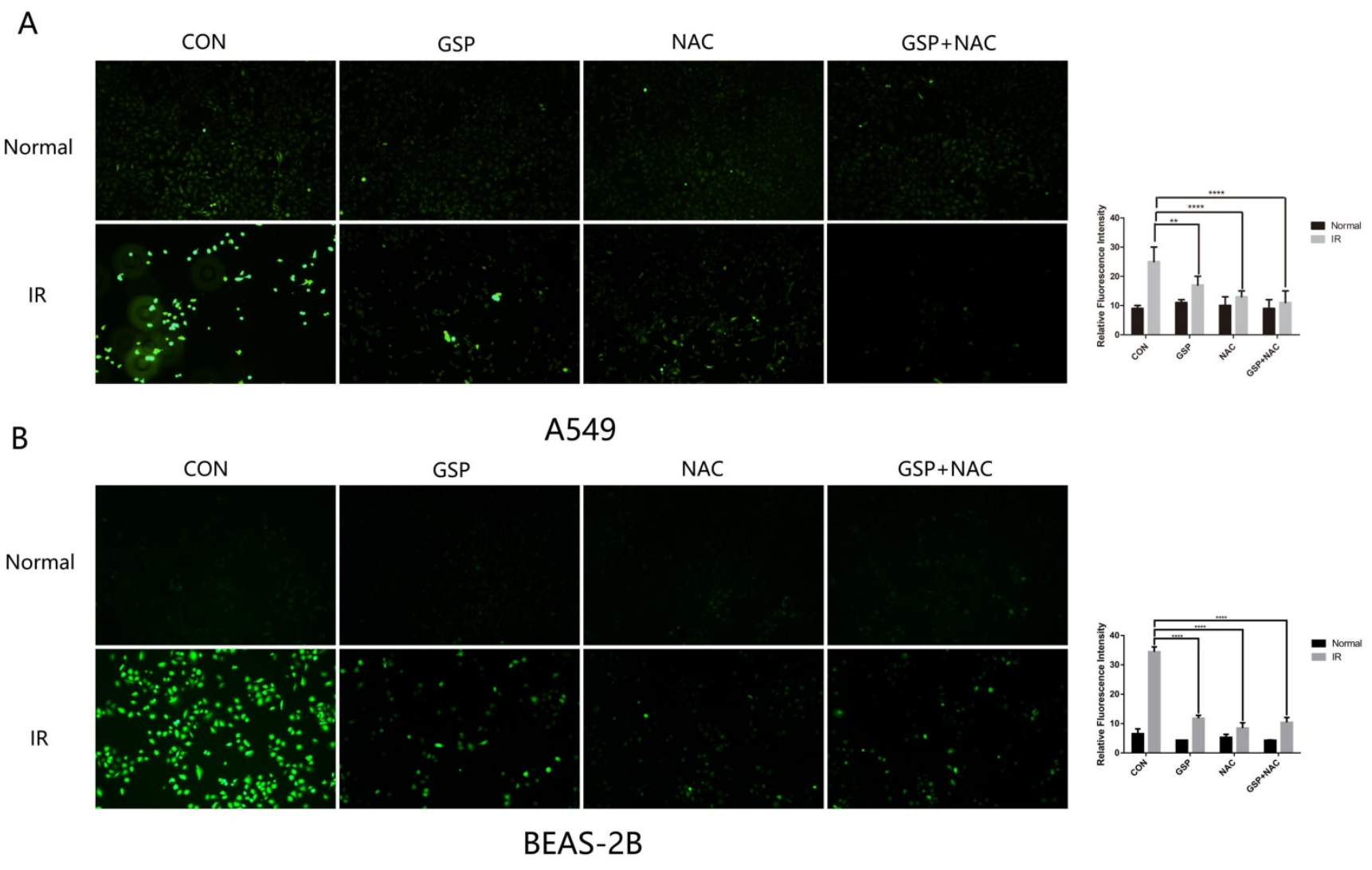

Figure 5. GSP reduces ROS levels in both cancer cells A549 and normal cells BEAS-2B. The anti-oxidant (NAC) was used as a positive control to detect the scavenging effect of GSP on free radicals after irradiation. One hour before irradiation $(8 \mathrm{~Gy}, 1 \mathrm{~Gy} / \mathrm{min})$, GSP were given at a concentration of $20 \mathrm{ug} / \mathrm{ml}$ and $\mathrm{NAC}$ at a concentration of $10 \mathrm{mmol} / \mathrm{L}$. The intracellular ROS level of A549 and BEAS-2B cells were detected after irradiation by Reactive Oxygen Species Fluorogenic Probe and were observed with confocal microscopy. GSP treatment significantly reduces ROS levels in both cancer cells A549 (A) and normal cells BEAS-2B (B). Data were presented as mean \pm SD ( $\mathrm{n}=3$ ). *P<0.05, **P $<0.01, * * * \mathrm{P}<0.001, * * * * \mathrm{P}<0.0001$. 

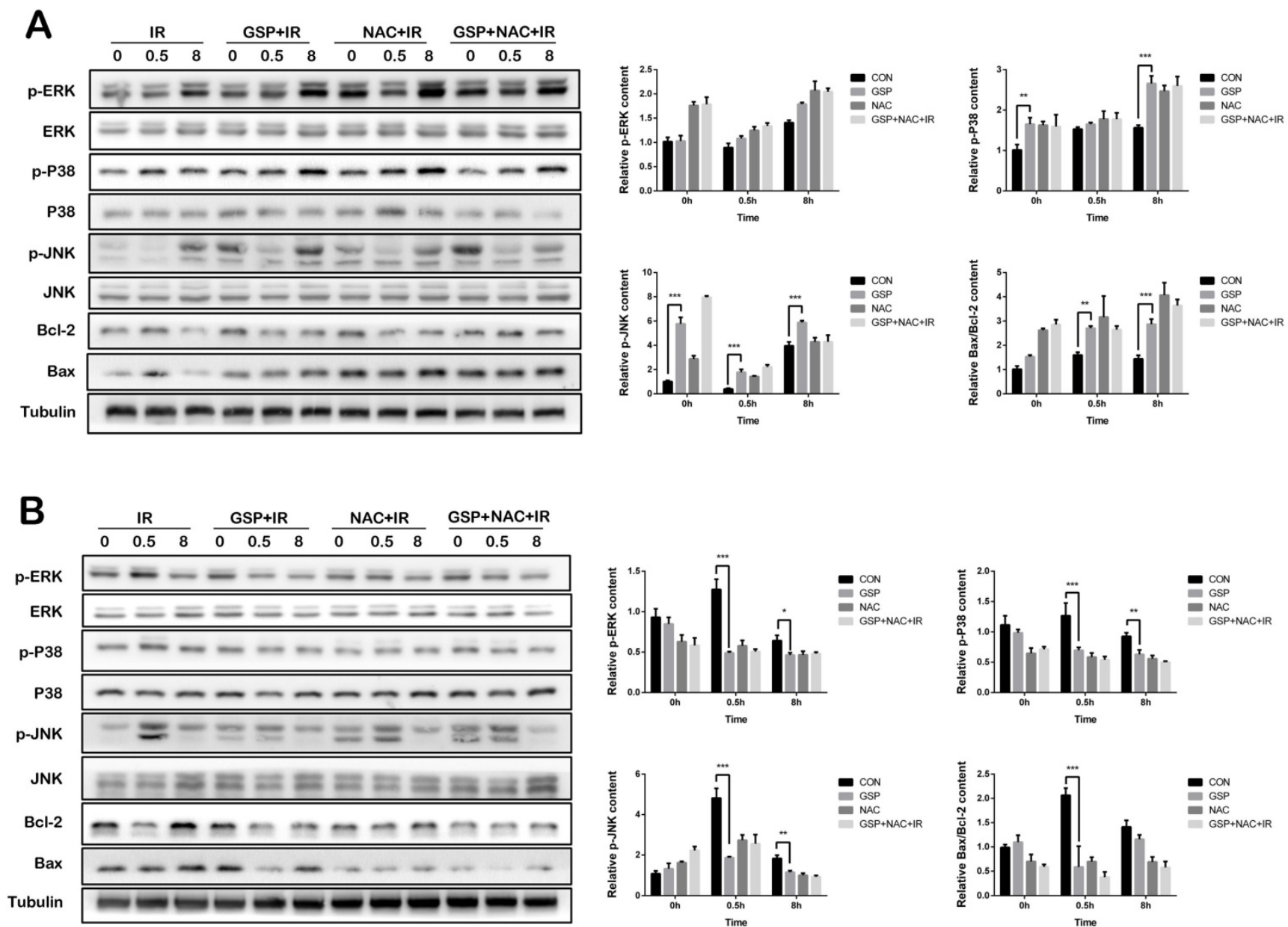

Figure 6. GSP differentially regulates MAPK signaling pathways in lung cancer cells and normal lung cells respectively. (A) In lung cancer cells A549, GSP significantly increases the expression of p-JNK, p-P38 protein before and after irradiation, and the value of Bax/Bcl-2 after irradiation, and has less effect on p-ERK protein. (B) In normal lung epithelial cells BEAS-2B, GSP reduces the expression of $\mathrm{p}-\mathrm{JNK}, \mathrm{p}-\mathrm{P} 38$, and $\mathrm{p}-\mathrm{ERK}$ proteins of MAPK family after irradiation, and reduces the expression of Bax and value of Bax/ Bcl-2. The raw density of Western blot figures was quantified. Data were presented as mean $\pm S D(n=3)$. $* P<0.05$. $* * P<0.01, * * * P<0.001, * * * * P<0.0001$.

Proanthocyanidins also have significant antiinflammatory effects. Proanthocyanidins can improve encephalitis [42, 43], Osteoarthritis [40,41] and skin inflammation [44]. The imbalance of Th1/Th2 was an important feature for the inflammatory response of the body. IL-6 is mainly secreted by Th2 cells or macrophages. IL-6 involved in RILI as a pro-inflammatory cytokine, and elevated serum IL-6 levels in patients can be used as independent predictors of clinical RILI [50]. In addition, increased serum IL-6 expression stimulates the growth of cancer cells [51]. IFN- $\gamma$ is mainly secreted by Th1 cells and induces cancer cells apoptosis by activating caspase- 8 and JNK-STAT [52]. IFN- $\gamma$ inhibits the metastasis and metabolism of cancer cells by reducing VEGF, TGF-a and other pro-angiogenic factors [53]. Our previous research results showed that GSP regulated the secretion of Th1/Th2-related cytokines in normal mice [12]. In this study, the GSP + IR group significantly inhibited IL-6 levels and increased IFN- $\gamma$ levels 12 hours after irradiation in lung cancer-bearing mice, suggesting that GSP played a role in mitigating inflammation and suppressing lung cancer.

The presence of reactive oxygen free radicals (ROS) caused by ionizing radiation was one of the important mechanisms causing the damage of cells and tissues. GSP, as a substance rich in phenolic hydroxyl, could rapidly neutralize a large amount of free radical oxygen ions and reduce radiation damage. In this study, we found that GSP significantly reduced ROS levels in normal cells BEAS-2B after irradiation. In cancer cells A549, GSP also reduced intracellular ROS levels, which may protect lung cancer cells against ionizing radiation to a certain extent. However, what we had observed was that GSP had a synergistic killing effect on tumor cells with radiotherapy. Therefore, the differential effect of GSP on normal cells and cancer cells does not work through its antioxidant capacity.

The mitogen-activated protein kinase (MAPK) is a specific type of silk/serine kinase, and composed of five parallel signaling pathways. The biological effects of each signal pathway are different due to their different distribution and activation degree in 

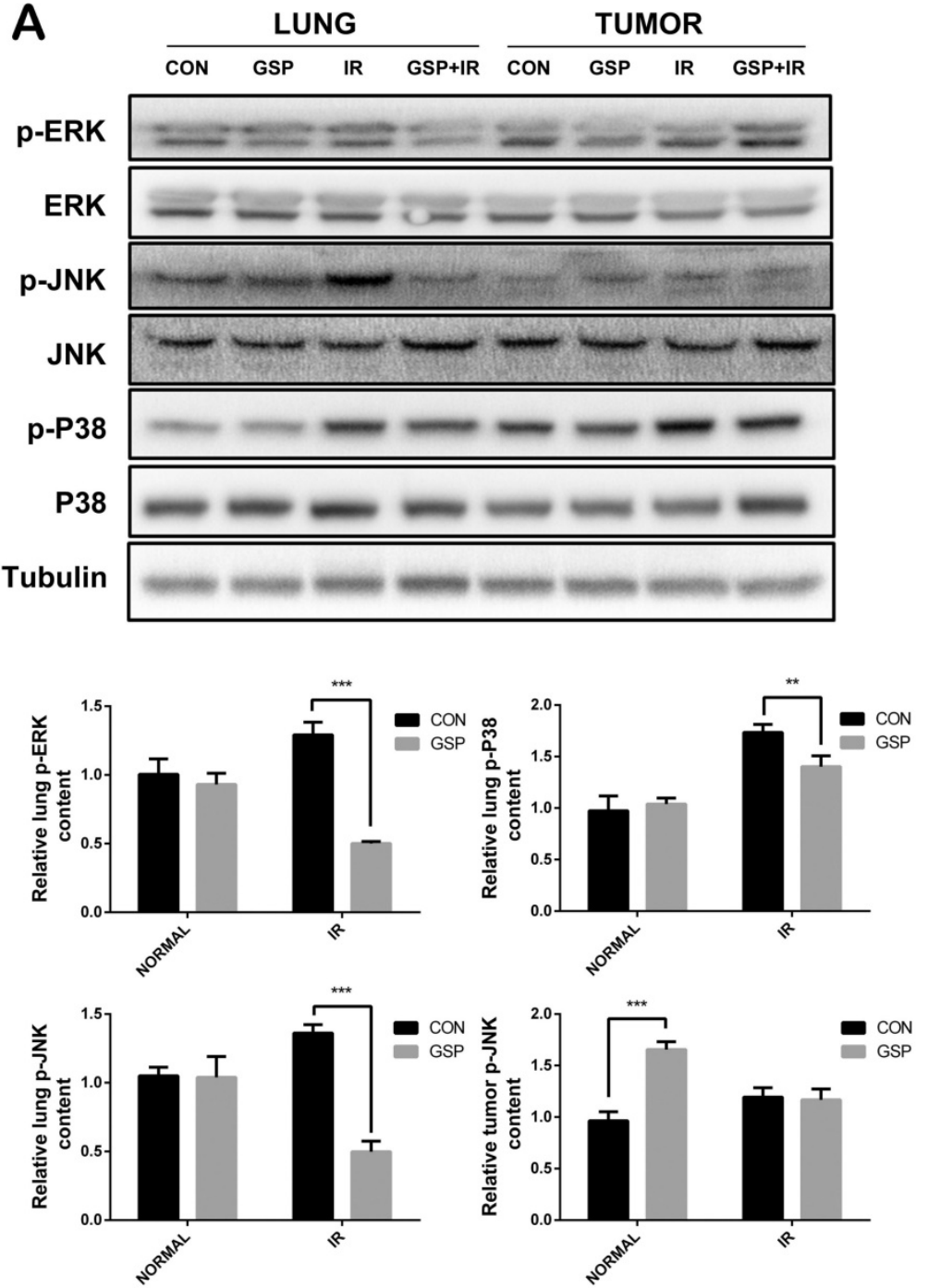

B

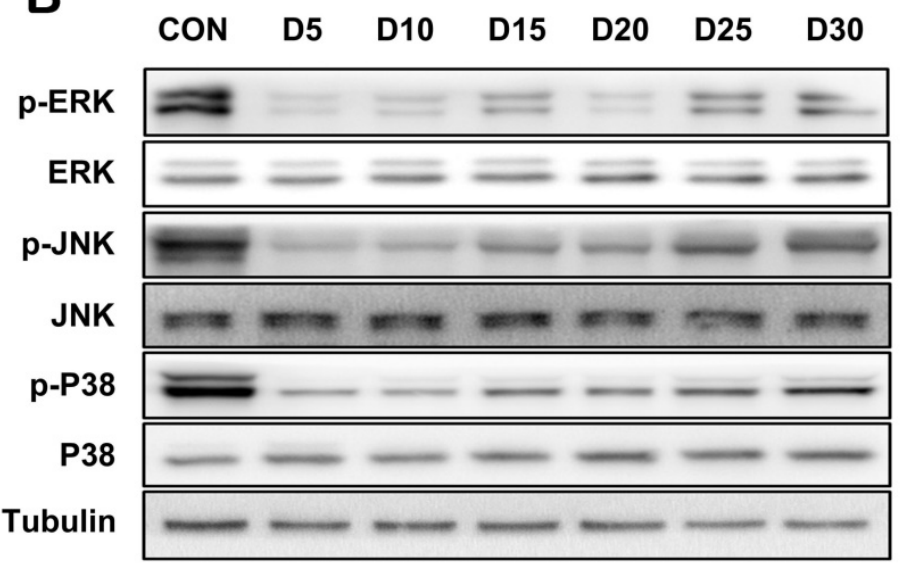

Figure 7. GSP differentially regulates MAPK signaling pathways in lung cancer tissues and normal lung tissues. (A) In tumor-bearing mice, ionizing radiation increases expression of $\mathrm{p}-\mathrm{JNK}, \mathrm{p}-\mathrm{P} 38$, and $\mathrm{p}$-ERK proteins of MAPK family in normal lung tissues, while GSP reduces the increments. In lung cancer tissues, GSP activates the expression of $\mathrm{p}-\mathrm{JNK}$ protein. (B) In normal mice, the expression of $\mathrm{p}-\mathrm{JNK}, \mathrm{p}-\mathrm{P} 38$, and $\mathrm{P}$-ERK proteins of MAPK family in the lung tissues is significantly reduced when GSP is given in diet, but the protein expression gradually increases over the feeding time. The raw density of Western blot figures was quantified. Data were presented as mean $\pm S D(n=3)$. $* P<0.05$. **P $<0.01$, ***P $<0.001$, **** $P<0.0001$.

different tumor cells. Ionizing radiation results in an increase in ROS, activating the expression of MAPK pathway [54], and increases the expression of inflammatory cytokines [55]. In addition, this study found that irradiation activated the MAPK signaling pathway. However, in normal cells BEAS-2B, GSP significantly inhibited the expression of p-JNK, p-ERK and p-P38, thereby protecting BEAS-2B cells against ionizing radiation. Proanthocyanidins have been reported to protect normal cells via down-regulating the MAPK signaling pathway. The study by Lee MH [44] has shown that proanthocyanidins-rich rose petal extract protects normal skin via down-regulating the MAPK signaling pathway. Proanthocyanidins also protect retinal pigment epithelial cells from UVB-induced damage by downregulating the MAPK signaling pathway [39]. However, in human lung cancer cells A549, GSP significantly enhanced the phosphorylation of JNK protein after irradiation, thereby enhancing the killing effect of radiotherapy on A549 cells in this study. JNK activation promotes apoptosis $[56,57]$ and autophagy [58] of cancer cells. Alnus hirsuta extract exerts anti-cancer activity against MCF-7 cells in vitro by activating JNK [31]. Bronchine D induces apoptosis and autophagy of lung cancer cell by up-regulating JNK phosphorylation levels in vitro and in vivo [34]. In contrast, down-regulating JNK phosphorylation promotes cancer cells survive [24].

\section{Conclusion}

This study showed that grape seed proanthocyanidins (GSP) had a radioprotective effect on normal lung tissue and a synergistic killing effect with radiotherapy on lung cancer tissues, which related to the differential regulation of the MAPK signaling pathway by GSP on normal lung and lung cancer. Moreover, GSP regulated secretion of cytokines IL-6 and IFN- $\gamma$ and expression of p53 and Ki67 on normal lung and lung cancer. Our findings suggest that GSP is expected to be an ideal radioprotective drug for lung cancer patients who are treated with radiotherapy. 


\section{Acknowledgements}

This work was supported in part by the grants from National Natural Science Foundation of China (No. 31670861, No. 81972968, No. 11635014, No. 31700739), Zhejiang Provincial Natural Science Foundation of China (No. LQ19H220001), Shanghai Science and Technology Talents Program Project (No. 18YF1429200) and Jiaxing Medical Key Discipline (No. 2019-fc-06).

\section{Competing Interests}

The authors have declared that no competing interest exists.

\section{References}

1. Miller KD, Nogueira L, Mariotto AB, et al. Cancer treatment and survivorship statistics, 2019. CA Cancer J Clin. 2019; 69(5): 363-385.

2. Stahl JM, Corso CD, Verma V, et al. Trends in stereotactic body radiation therapy for stage I small cell lung cancer. Lung Cancer. 2017; 103: 11-16.

3. Jin H, Tucker SL, Liu HH, et al. Dose-volume thresholds and smoking status for the risk of treatment-related pneumonitis in inoperable non-small cell lung cancer treated with definitive radiotherapy. Radiother Oncol. 2009; 91(3): 427-32.

4. Kong FM, Hayman JA, Griffith KA, et al. Final toxicity results of a radiation-dose escalation study in patients with non-small-cell lung cancer (NSCLC): predictors for radiation pneumonitis and fibrosis. Int J Radiat Oncol Biol Phys. 2006; 65(4): 1075-86.

5. Mahmood J, Jelveh S, Calveley V, et al. Mitigation of radiation-induced lung injury by genistein and EUK-207. Int J Radiat Biol. 2011; 87(8): 889-901.

6. Lee JC, Kinniry PA, Arguiri E, et al. Dietary curcumin increases antioxidant defenses in lung, ameliorates radiation-induced pulmonary fibrosis, and improves survival in mice. Radiat Res. 2010; 173(5): 590-601.

7. Robb WB, Condron C, Moriarty $\mathrm{M}$, et al. Taurine attenuates radiation-induced lung fibrosis in C57/Bl6 fibrosis prone mice. Ir J Med Sci. 2010; 179(1): 99-105.

8. Liu $\mathrm{Y}, \mathrm{Yu} \mathrm{H}$, Zhang $\mathrm{C}$, et al. Protective effects of berberine on radiationinduced lung injury via intercellular adhesion molecular-1 and transforming growth factor-beta-1 in patients with lung cancer. Eur J Cancer. 2008; 44(16): 2425-32.

9. Bagchi $\mathrm{D}$, Bagchi $\mathrm{M}$, Stohs SJ, et al. Free radicals and grape seed proanthocyanidin extract: importance in human health and disease prevention. Toxicology. 2000; 148(2-3): 187-97.

10. Bagchi D, Garg A, Krohn RL, et al. Oxygen free radical scavenging abilities of vitamins $\mathrm{C}$ and $\mathrm{E}$, and a grape seed proanthocyanidin extract in vitro. Res Commun Mol Pathol Pharmacol. 1997; 95(2): 179-89.

11. Huang Y, Zhao H, Cao K, et al. Radioprotective Effect of Grape Seed Proanthocyanidins In vitro and In vivo. Oxid Med Cell Longev. 2016; 2016: 5706751.

12. Huang $\mathrm{Y}$, Liu $\mathrm{W}$, Liu $\mathrm{H}$, et al. Grape seed pro-anthocyanidins ameliorates radiation-induced lung injury. J Cell Mol Med. 2014; 18(7): 1267-77.

13. Ma X and S Ning. Cyanidin-3-glucoside attenuates the angiogenesis of breast cancer via inhibiting STAT3/VEGF pathway. Phytother Res. 2019; 33(1): 81-89.

14. De SM, Sun X, Peluzio M, et al. Anthocyanins/anthocyanidins and colorectal cancer: What is behind the scenes? Crit Rev Food Sci Nutr. 2019; 59(1): 59-71.

15. Abbasi BH, Nazir M, Muhammad W, et al. A Comparative Evaluation of the Antiproliferative Activity against HepG2 Liver Carcinoma Cells of Plant-Derived Silver Nanoparticles from Basil Extracts with Contrasting Anthocyanin Contents. Biomolecules. 2019; 9(8): 320.

16. Ribera-Fonseca A, Jimenez D, Leal P, et al. The Anti-Proliferative and Anti-Invasive Effect of Leaf Extracts of Blueberry Plants Treated with Methyl Jasmonate on Human Gastric Cancer In vitro Is Related to Their Antioxidant Properties. Antioxidants (Basel). 2020; 9(1): 45.

17. Yue E, Tuguzbaeva G, Chen X, et al. Anthocyanin is involved in the activation of pyroptosis in oral squamous cell carcinoma. Phytomedicine. 2019; 56: 286-294

18. Rossi M, Strikoudi P, Spei ME, et al. Flavonoids and bladder cancer risk. Cancer Causes Control. 2019; 30(5): 527-535.

19. Sajadimajd S, Bahramsoltani R, Iranpanah A, et al. Advances on Natural Polyphenols as Anticancer Agents for Skin Cancer. Pharmacol Res. 2020; 151: 104584

20. Liu X, Zhang D, Hao Y, et al. Cyanidin Curtails Renal Cell Carcinoma Tumorigenesis. Cell Physiol Biochem. 2018; 46(6): 2517-2531.

21. Tyagi A, Kumar S, Raina K, et al. Differential effect of grape seed extract and its active constituent procyanidin B2 3,3"-di-O-gallate against prostate cancer stem cells. Mol Carcinog. 2019; 58(7): 1105-1117.

22. Sharma SD, Meeran SM, and Katiyar SK. Proanthocyanidins inhibit in vitro and in vivo growth of human non-small cell lung cancer cells by inhibiting the prostaglandin $\mathrm{E}(2)$ and prostaglandin $\mathrm{E}(2)$ receptors. Mol Cancer Ther. 2010; 9(3): 569-80.

23. Guo YJ, Pan WW, Liu SB, et al. ERK/MAPK signalling pathway and tumorigenesis. Exp Ther Med. 2020; 19(3): 1997-2007.

24. Lv C, Fu S, Dong Q, et al. PAGE4 promotes prostate cancer cells survive under oxidative stress through modulating MAPK/JNK/ERK pathway. J Exp Clin Cancer Res. 2019; 38(1): 24

25. Che Y, Li Y, Zheng F, et al. TRIP4 promotes tumor growth and metastasis and regulates radiosensitivity of cervical cancer by activating MAPK, PI3K/AKT, and hTERT signaling. Cancer Lett. 2019; 452: 1-13.

26. Chen $\mathrm{H}$, Chen $\mathrm{W}$, Zhang $\mathrm{X}$, et al. E26 transformation (ETS)specific related transcription factor3 (ELF3) orchestrates a positive feedback loop that constitutively activates the MAPK/Erk pathway to drive thyroid cancer. Oncol Rep. 2019; 41(1): 570-578.

27. Peng WX, Huang JG, Yang L, et al. Linc-RoR promotes MAPK/ERK signaling and confers estrogen-independent growth of breast cancer. Mol Cancer. 2017; 16(1): 161

28. Wang B, Pan LY, Kang N, et al. PP4R1 interacts with HMGA2 to promote non-small-cell lung cancer migration and metastasis via activating MAPK/ ERK-induced epithelial-mesenchymal transition. Mol Carcinog. 2020; 59(5): $467-477$

29. Lim W, Jeong W, and Song G. Delphinidin suppresses proliferation and migration of human ovarian clear cell carcinoma cells through blocking AKT and ERK1/2 MAPK signaling pathways. Mol Cell Endocrinol. 2016; 422: 172-181.

30. Li S, Fong KW, Gritsina G, et al. Activation of MAPK Signaling by CXCR7 Leads to Enzalutamide Resistance in Prostate Cancer. Cancer Res. 2019; 79(10): 5800-2592.

31. Ryu M, Sung CK, Im YJ, et al. Activation of JNK and p38 in MCF-7 Cells and the In vitro Anticancer Activity of Alnus hirsuta Extract. Molecules. 2020; 25(5): 1073.

32. Zhang Y, Yang WK, Wen GM, et al. High expression of PRKDC promotes breast cancer cell growth via p38 MAPK signaling and is associated with poor survival. Mol Genet Genomic Med. 2019; 7(11): 908.

33. He W, Cao P, Xia Y, et al. Potent inhibition of gastric cancer cells by a natural compound via inhibiting TrxR1 activity and activating ROS-mediated p38 MAPK pathway. Free Radic Res. 2019; 53(1): 104-114.

34. Fan J, Ren D, Wang J, et al. Bruceine D induces lung cancer cell apoptosis and autophagy via the ROS/MAPK signaling pathway in vitro and in vivo. Cell Death Dis. 2020; 11(2): 126.

35. Shin DY, Lee WS, Lu JN, et al. Induction of apoptosis in human colon cancer HCT-116 cells by anthocyanins through suppression of Akt and activation of p38-MAPK. Int J Oncol. 2009; 35(6): 1499-504

36. Feng R, Ni HM, Wang SY, et al. Cyanidin-3-rutinoside, a natural polyphenol antioxidant, selectively kills leukemic cells by induction of oxidative stress. J Biol Chem. 2007; 282(18): 13468-76.

37. Mantena SK and Katiyar SK. Grape seed proanthocyanidins inhibit UVradiation-induced oxidative stress and activation of MAPK and NF-kappaB signaling in human epidermal keratinocytes. Free Radic Biol Med. 2006; 40(9): $1603-14$

38. Sharma SD, Meeran SM, and Katiyar SK. Dietary grape seed proanthocyanidins inhibit UVB-induced oxidative stress and activation of mitogen-activated protein kinases and nuclear factor-kappaB signaling in in vivo SKH-1 hairless mice. Mol Cancer Ther. 2007; 6(3): 995-1005.

39. Silvan JM, Reguero $\mathrm{M}$, and Pascual-Teresa S. A protective effect of anthocyanins and xanthophylls on UVB-induced damage in retinal pigment epithelial cells. Food Funct. 2016; 7(2): 1067-76

40. Wongwichai $T$, Eeyakasem $\mathrm{P}$, Pruksakorn $\mathrm{D}$, et al Anthocyanins and metabolites from purple rice inhibit IL-1beta-induced matrix metalloproteinases expression in human articular chondrocytes through the NFkappaB and ERK/MAPK pathway. Biomed Pharmacother. 2019; 112: 108610.

41. Sun $Y$ and Li L. Cyanidin-3-glucoside inhibits inflammatory activities in human fibroblast-like synoviocytes and in mice with collagen-induced arthritis. Clin Exp Pharmacol Physiol. 2018; 45(10): 1038-1045.

42. Li J, Shi Z, and Mi Y. Purple sweet potato color attenuates high fat-induced neuroinflammation in mouse brain by inhibiting MAPK and NF-kappaB activation. Mol Med Rep. 2018; 17(3): 4823-4831.

43. Zhang Y, Meng Q, Yin J, et al. Anthocyanins attenuate neuroinflammation through the suppression of MLK3 activation in a mouse model of perioperative neurocognitive disorders. Brain Res. 2020; 1726: 146504.

44. Lee MH, Nam TG, Lee I, et al. Skin anti-inflammatory activity of rose petal extract (Rosa gallica) through reduction of MAPK signaling pathway. Food Sci Nutr. 2018; 6(8): 2560-2567.

45. Cao K, Lei X, Liu H, et al. Polydatin alleviated radiation-induced lung injury through activation of Sirt3 and inhibition of epithelial-mesenchymal transition. J Cell Mol Med. 2017; 21(12): 3264-3276.

46. Li K, Zhang $\mathrm{M}$, Chen $\mathrm{H}$, et al. Anthocyanins from black peanut skin protect against UV-B induced keratinocyte cell and skin oxidative damage through activating Nrf 2 signaling. Food Funct. 2019; 10(10): 6815-6828.

47. Wei J, Wu H, Zhang $\mathrm{H}$, et al. Anthocyanins inhibit high glucose-induced renal tubular cell apoptosis caused by oxidative stress in $\mathrm{db} / \mathrm{db}$ mice. Int J Mol Med. 2018; 41(3): 1608-1618

48. Martin J, Diaz-Montana EJ, and Asuero AG. Recovery of Anthocyanins Using Membrane Technologies: A Review. Crit Rev Anal Chem. 2018; 48(3): 143-175. 
49. Ongkowijoyo P, Luna-Vital DA, and Gonzalez ME. Extraction techniques and analysis of anthocyanins from food sources by mass spectrometry: An update. Food Chem. 2018; 250: 113-126.

50. Huang Y, Zhang W, Yu F, et al. The Cellular and Molecular Mechanism of Radiation-Induced Lung Injury. Med Sci Monit. 2017; 23: 3446-3450.

51. Hong SH, Ondrey FG, Avis IM, et al. Cyclooxygenase regulates human oropharyngeal carcinomas via the proinflammatory cytokine IL-6: a general role for inflammation? FASEB J. 2000; 14(11): 1499-507.

52. Stark GR and Darnell JE. The JAK-STAT pathway at twenty. Immunity. 2012; 36(4): 503-14.

53. Ni C, Ma P, Qu L, et al. Accelerated tumour metastasis due to interferongamma receptor-mediated dissociation of perivascular cells from blood vessels. J Pathol. 2017; 242(3): 334-346.

54. Afaq F, Malik A, Syed D, et al. Pomegranate fruit extract modulates UV-B-mediated phosphorylation of mitogen-activated protein kinases and activation of nuclear factor kappa B in normal human epidermal keratinocytes paragraph sign. Photochem Photobiol. 2005; 81(1): 38-45.

55. Wang J, Huang J, Wang L, et al. Urban particulate matter triggers lung inflammation via the ROS-MAPK-NF-kappaB signaling pathway. J Thorac Dis. 2017; 9(11): 4398-4412.

56. Smith WE, Kane AV, Campbell ST, et al. Shiga toxin 1 triggers a ribotoxic stress response leading to p38 and JNK activation and induction of apoptosis in intestinal epithelial cells. Infect Immun. 2003; 71(3): 1497-504.

57. Chang $\mathrm{C}$, Zhu YQ, Mei JJ, et al. Involvement of mitochondrial pathway in NCTD-induced cytotoxicity in human hepG2 cells. J Exp Clin Cancer Res. 2010; 29(1): 145

58. Wu RW, Yow CM, Wong CK, et al. Photodynamic therapy (PDT) - Initiation of apoptosis via activation of stress-activated p38 MAPK and JNK signal pathway in H460 cell lines. Photodiagnosis Photodyn Ther. 2011; 8(3): 254-63. 